Article

\title{
Early Observations of the Interstellar Comet 2I/Borisov
}

\author{
Chien-Hsiu Lee ${ }^{\mathbb{D}}$ \\ NSF's National Optical-Infrared Astronomy Research Laboratory, Tucson, AZ 85719, USA; lee@noao.edu; \\ Tel.: +1-520-318-8368
}

Received: 26 November 2019; Accepted: 11 December 2019; Published: 17 December 2019

\begin{abstract}
I/Borisov is the second ever interstellar object (ISO). It is very different from the first ISO 'Oumuamua by showing cometary activities, and hence provides a unique opportunity to study comets that are formed around other stars. Here we present early imaging and spectroscopic follow-ups to study its properties, which reveal an (up to) $5.9 \mathrm{~km}$ comet with an extended coma and a short tail. Our spectroscopic data do not reveal any emission lines between 4000-9000 Angstrom; nevertheless, we are able to put an upper limit on the flux of the $\mathrm{C} 2$ emission line, suggesting modest cometary activities at early epochs. These properties are similar to comets in the solar system, and suggest that 2I/Borisov-while from another star-is not too different from its solar siblings.
\end{abstract}

Keywords: comets: general; comets: individual (2I/Borisov); solar system: formation

\section{Introduction}

2I/Borisov was first seen by Gennady Borisov on 30 August 2019. As more observations were conducted in the next few days, there was growing evidence that this might be an interstellar object (ISO), especially its large orbital eccentricity. However, the first astrometric measurements do not have enough timespan and are not of same quality, hence the high eccentricity is yet to be confirmed. This had all changed by 11 September; where more than 100 astrometric measurements over 12 days, Ref [1] pinned down the orbit elements of 2I/Borisov, with an eccentricity of $3.15 \pm 0.13$, hence confirming the interstellar nature. For comparison, the orbital elements of 2I/Borisov imply a hyperbolic excess speed of $30 \mathrm{~km} / \mathrm{s}$, comparable to and slightly larger than 'Oumuamua [2], whose excess speed was $26 \mathrm{~km} / \mathrm{s}$. More interestingly (and unlike 'Oumuamua), 2I/Borisov showed an extended coma and a broad, short tail $\left(\sim 15^{\prime \prime}\right)$ in short $\mathrm{g}^{\prime}$ - and $\mathrm{r}^{\prime}$-band exposures taken on 10 September 2019 [1]. Thus, it is clear that this second-known interstellar interloper is a comet.

It is known that comets in our solar system can serve as a reservoir of the primordial materials of the proto-planetary disk when the planets were formed and as the disk cooled down. Hence, the interstellar comet 2I/Borisov provides a unique opportunity to probe chemical compositions of other exo-planetary systems. We note that unlike 'Oumuamua, 2I/Borisov was spotted very early on. It was at a distance of $\sim 3$ a.u. on 30 August, and the existence of a short tail indicates that its cometary activities just started, and we can still probe the pristine material on its surface. We expect, as this interstellar comet is on its way in (perihelion at $\sim 2$ a.u.), that the tail will develop further. Here we present early observations, at the epoch of 17 September 2019 UT.

\section{Observation}

From JPL/HORIZONS, it was predicted that 2I/Borisov could brighten to $\mathrm{V}=18.5$ mag around September 2019. We were not only aiming at the comet nucleus, but also the cometary tail as well, which can be much fainter than the nucleus. Hence, we conducted imaging observations with a total of 600-second integrations in the V-band with the Alhambra Faint Object Spectrograph and Camera (ALFOSC) mounted on the $2.5 \mathrm{~m}$ Nordic Optical Telescope. In 600-second integration time, ALFOSC 
can reach to $\mathrm{V}=22 \mathrm{mag}$ with a signal-to-noise ratio $(\mathrm{S} / \mathrm{N}) \sim 20$. The field of view $(\mathrm{FoV})$ of ALFOSC is $6.4 \times 6.4$ squared arcminutes, sampled by $2048 \times 2048$ pixels in the imaging mode, translating into $0.2138 \mathrm{arcsec} /$ pixel. To better remove CCD artifacts and cosmic rays, we divided the total 600-second integration into $3 \times 200$-second single exposures, each of them reaching to $\mathrm{S} / \mathrm{N} \sim 10$ at $\mathrm{V}=22 \mathrm{mag}$. Even in the single exposure, 2I/Borisov was bright enough to allow us to align and stack individual exposures into a deeper stack.

In addition to imaging, we also performed spectroscopic observations to search for emission lines that can be indicative of outgassing molecules of 2I/Borisov. We employed the $\mathrm{g} 4 \mathrm{grism}$ of ALFOSC, to cover the C2 emission lines (4700 Ang. $<\lambda<6000$ Ang.) and potential absorption features from hydrated silicates at 7000 Ang. With 10-minute integration time using a 2.5-arcsecond slit under a median sky condition (1.5-arcsecond seeing condition under 1.5 airmass during grey nights), we expect to reach a $\mathrm{S} / \mathrm{N} \sim 20$ of the continuum. This sufficiently allows us to detect the above-mentioned features in the spectra. The slit was aligned to a fixed position angle (90 degrees, east of north) with atmospheric dispersion corrector to correct for atmospheric differential refraction. To enable the removal of cosmic rays and CCD artifacts, we thus divided the $600 \mathrm{~s}$ integration into $3 \times 200$-second single exposures.

The above-mentioned observations, both photometrically and spectroscopically, were conducted on 17 September 2019 UT, between 5 and 6 a.m. Data reduction and analysis were performed in a standard fashion, making use of the Image Reduction and Analysis Facility (IRAF). The photometric observations were de-trended with bias subtraction and flat fielding. After de-trending, we further aligned the three individual images and median combined them into a deeper image. The spectra were also reduced with corresponding bias subtraction, flat fielding, and wavelength-calibration against ThAr lamp. To remove cosmic ray and CCD artifacts, the three individual spectra were median combined into a single, deeper spectrum.

\section{Result and Discussion}

Our deep imaging (Figure 1) clearly detects 2I/Borisov, with an extended coma (compared to field stars, see Figure 1) and a short tail, spanning $\sim 15$ arcsec in anti-solar direction (with a position angle of $\sim 300$ degrees). To extract the photometry from the imaging, we used SExtractor [3]. Adopting a circular aperture with a radius of 20 arcseconds and calibrated using field stars against the APASS survey, we estimated the comet to be $V=18.93 \pm 0.03$ mag. Given the brightness, we can estimate the size of the comet as follows. We computed the absolute magnitudes $H_{V}$ using

$$
H_{V}=m_{V}-5 \log _{10}\left(r_{H} \Delta\right)-\Phi(\alpha)
$$

where $r_{H}$ and $\Delta$ are the helio- and geo-centric distances (in a.u.) respectively. $\Phi(\alpha)$ is the phase function given a phase angle $\alpha$. We assume $\Phi(\alpha)=\beta \alpha$ and $\beta=0.04 \mathrm{mag} /$ degree. With the absolute magnitude in hand, we can further calculate the effective scattering cross-section $C_{e}$ by

$$
C_{e}=\frac{1.5 \times 10^{6}}{p_{V}} \times 10^{-0.4 H_{V}} \text {, }
$$

where $p_{V}$ corresponds to the V-band geometric albedo. As the albedo of 2I/Borisov is yet to be determined, here we assume $p_{V}=0.05$ [4] for the comet nuclei, which is similar to the albedo distribution of primitive class asteroids. Using the above-mentioned equations, the mean magnitude corresponds to $C_{e}=108 \mathrm{~km}^{2}$. This translates into an equal-area circle of radius $\sqrt{\frac{C_{e}}{\pi}}=5.9 \mathrm{~km}$; assuming a nominal density $\rho=0.5 \times 10^{3} \mathrm{~kg} / \mathrm{m}^{3}$ from the Rosetta observations of comet Churyumov-Gerasimenko [5], we estimate the mass of $2 \mathrm{I} /$ Borisov to be $2 \times 10^{14} \mathrm{~kg}$. We should note that the photometry is affected by the extended coma, and hence the estimates (both size and mass) should be treated as an upper limit. Nevertheless, even with this upper limit, the size and the 
mass of 2I/Borisov are in similar ranges of the comets in the solar system [4,6]. We note that our independent observations lead to the results similar to [1,7-9].
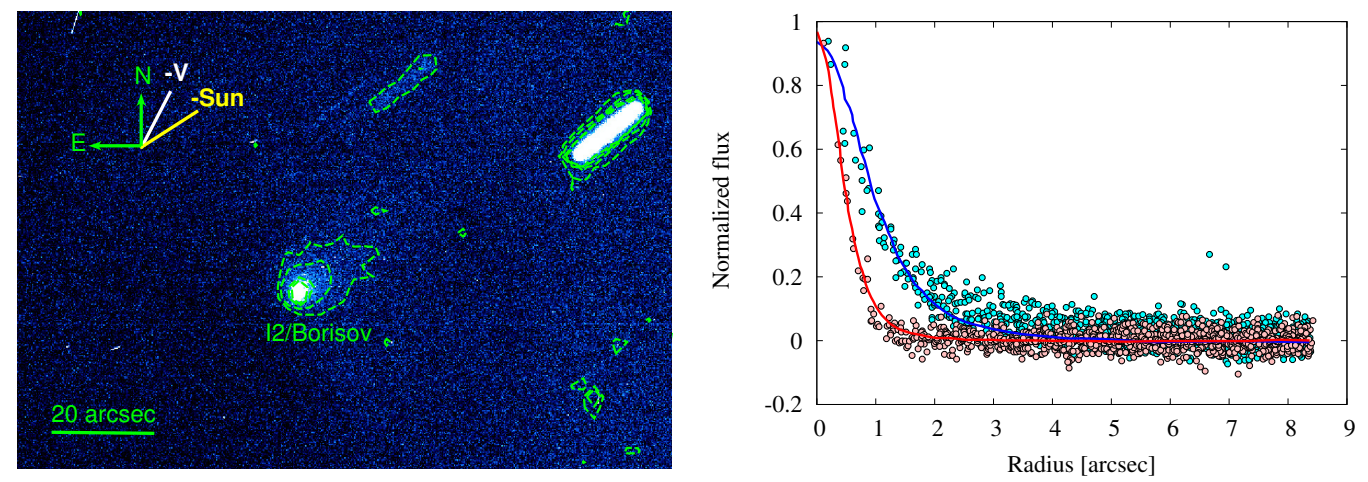

Figure 1. Left: V-band imaging of 2I/Borisov. The direction of the velocity and the Sun, and the North (to the up) and East (to the left) are marked accordingly. The contours indicate the appearance of an extend coma. Right: brightness profile of 2I/Borisov (cyan filled circles) and a field star taken under sidereal tracking (pink filled circles). The blue and red lines indicate the best-fit point-spread-function. The brightness profile of 2I/Borisov significantly different from a point-source (field star), suggesting the existence of an extend coma.

It has been reported that the reflectance spectrum of 2I/Borisov is similar to that of comets and D-type asteroids seen in the solar system [10]. However, in our spectrum there is no sign of emission lines, especially C2 (4700 Ang. $<\lambda<6000$ Ang.), which are prominent features seen in the comets in the solar system. We employ a procedure similar to that of [11] to obtain an upper limit from the non-detection of the $\mathrm{C} 2$ lines. We first calibrate the spectral continuum using the broad-band photometry. As we only have V-band ( $\lambda_{c}=5500$ Ang.) in hand, we thus focus on the C2 emission lines. On 17 September 2019, we measured V = 18.93 magnitude for 2I/Borisov. Since a V= 0 (Vega) star has a flux density $f_{\lambda}=3.75 \times 10^{-9} \mathrm{erg} / \mathrm{cm}^{2} / \mathrm{s} /$ Ang. [12], we can infer the mean V-band continuum flux density of 2I/Borisov to be $f_{V}=1.0 \times 10^{-16} \mathrm{erg} / \mathrm{cm}^{2} / \mathrm{s} /$ Ang. The strong $\mathrm{C} 2 \Delta \mathrm{V}=0$ band is confined in the wavelength range of 5050-5220 Ang. [13], yet there is no detection in our spectrum of $2 \mathrm{I} /$ Borisov. To quantify the level of non-detection, we made use of the continuum adjacent to the $\mathrm{C} 2 \Delta \mathrm{V}$ $=0$ band with equal width, both blueward and redward at the corresponding wavelength ranges of $B_{C}$ at 4880 Ang. $<\lambda<5049$ Ang. and $R_{C}$ at 5221 Ang. $<\lambda<5390$ Ang., respectively (as marked in Figure 2). We estimated uncertainties statically (expressed in terms of fractions of the continuum) of $B_{C}, C 2$, and $R_{C}$ as $0.17,0.19$, and 0.18 , respectively. We used the largest uncertainty among the three, i.e., $1 \sigma=0.19$, as the fractional uncertainty in the continuum in the $\mathrm{C} 2 \Delta \mathrm{V}$-band. This provided an empirical upper limit of the gas flux density $f_{\mathrm{C} 2}=0.57 \times f_{V}=5.7 \times 10^{-17} \mathrm{erg} / \mathrm{cm}^{2} / \mathrm{s} /$ Ang. at $3 \sigma$-level. By summing up the flux in the $\Delta \lambda=170 \mathrm{Ang}$. of the $\mathrm{C} 2 \Delta \mathrm{V}$-band, we further estimated an upper limit of the flux $F=f_{\mathrm{C} 2} \times \Delta \lambda<9.7 \times 10^{-15} \mathrm{erg} / \mathrm{cm}^{2} / \mathrm{s}$ at $3 \sigma$-level. We note the non-detection of $\mathrm{C} 2$ lines could be due to our shallow spectrum, or because the comet is at lower activity at this epoch; the surface activity could increase significantly as it approaches perihelion. We note that our independent spectroscopic observations lead to the results similar to $[7,14,15]$. 


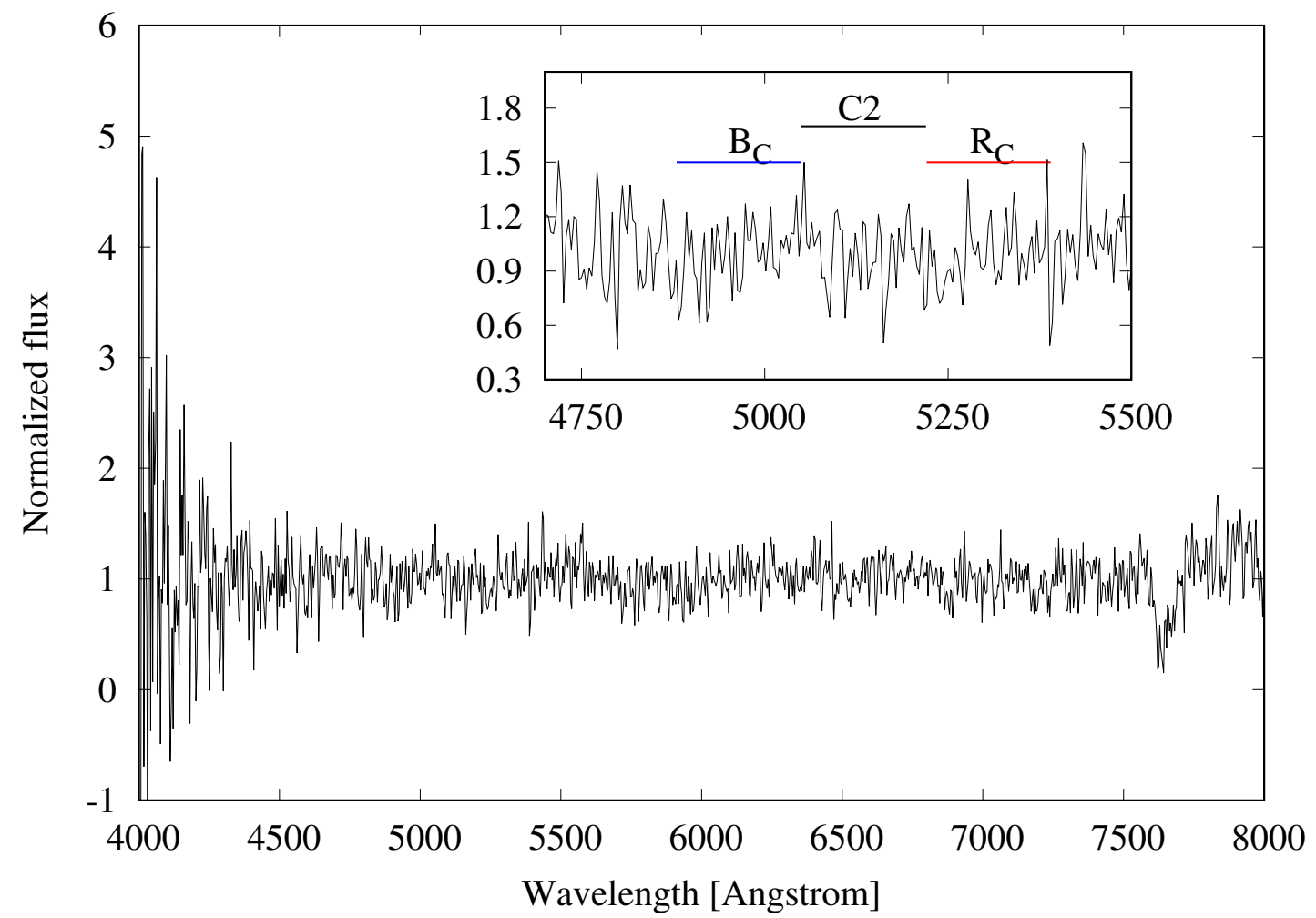

Figure 2. Normalized optical spectrum of 2I/Borisov. There are no traces of strong emission lines. Inset: Normalized spectrum of $2 \mathrm{I} /$ Borisov at the wavelength of $\mathrm{C} 2 \Delta \mathrm{V}=0$ emission (5050 Ang. $<\lambda<5220$ Ang.). The horizontal lines mark the wavelengths of the $\mathrm{C} 2$ band and the blue and red continua $\left(B_{C}\right.$ and $\left.R_{C}\right)$ used to estimate the noise.

To summarize, both imaging and spectroscopy show that 2I/Borisov shares similar properties with comets in the solar system, hence it is unremarkable except for it being ejected from another star. However, we should note that comet ejection is not uncommon, and can happen in the solar system due to the migration of giant planets, as predicted by the Nice model [16-18]. Current astrometric data indicate that 2I/Borisov came from and will return to a radiant close to the direction of open star cluster Stock 2 [10]. However, we should note that given its speed $(\sim 30 \mathrm{~km} / \mathrm{s})$, Stock 2 would not be at its current location and could not have been the origin of 2I/Borisov after it travelled $\sim 33,000$ years. We will be able to better determine the trajectory of 2I/Borisov after it passes perihelion; combining with the exquisite astrometry of nearby stars with Gaia, we will be able to pin down its home star. Furthermore, with LSST on the horizon, we will be able to identify incoming interstellar objects early on; this will enable fly-by or sample-return missions, e.g., comet interceptors, to provide in situ investigations of interstellar objects. Another possibility is that some of the interstellar objects (comets and asteroids) can become trapped in the solar system by three-body gravitational interactions involving Jupiter and the Sun (Lingam \& Loeb 2018). Hence, it would be possible to carry out in situ explorations in more detail.

Funding: This research received no external funding.

Acknowledgments: We are indebted to the Nordic Optical Telescope staff, especially Anlaug Amanda Djupvik, for carrying out the observations under the fast-track service program. This work is based on observations made with the Nordic Optical Telescope, operated by the Nordic Optical Telescope Scientific Association at the Observatorio del Roque de los Muchachos, La Palma, Spain, of the Instituto de Astrofisica de Canarias. The data presented here were obtained with ALFOSC, which is provided by the Instituto de Astrofisica de Andalucía (IAA) under a joint agreement with the University of Copenhagen and NOTSA.

Conflicts of Interest: The author declares no conflict of interest. 


\section{References}

1. Guzik, P.; Drahus, M.; Rusek, K.; Waniak, W.; Cannizzaro, G.; Pastor-Marazuela, I. Initial characterization of interstellar comet 2I/Borisov. arXiv 2019, arXiv:1909.05851.

2. Meech, K.J.; Weryk, R.; Micheli, M.; Kleyna, J.T.; Hainaut, O.R.; Jedicke, R.; Wainscoat, R.J.; Chambers, K.C.; Keane, J.V.; Petric, A.; et al. A brief visit from a red and extremely elongated interstellar asteroid. Nature 2017, 552, 378. [CrossRef] [PubMed]

3. Bertin, E.; Arnouts, S. SExtractor: Software for source extraction. Astron. Astrophys. Suppl. Ser. 1996, 117, 393. [CrossRef]

4. Fernández, Y.R.; Kelley, M.S.; Lamy, P.L.; Toth, I.; Groussin, O.; Lisse, C.M.; A'Hearn, M.F.; Bauer, J.M.; Campins, H.; Fitzsimmons, A.; et al. Thermal properties, sizes, and size distribution of Jupiter-family cometary nuclei. Icarus 2013, 226, 1138. [CrossRef]

5. Jorda, L.; Gaskell, R.; Capanna, C.; Hviid, S.; Lamy, P.; Ďurech, J.; Faury, G.; Groussin, O.; Gutiérrez, P.; Jackman, C.; et al. The global shape, density and rotation of Comet 67P/Churyumov-Gerasimenko from preperihelion Rosetta/OSIRIS observations. Icarus 2016, 277, 257. [CrossRef]

6. Britt, D.T.; Consolmagno, G.J.; Merline, W.J. Small body density and porosity: New data, new insights. In Proceedings of the 37th Annual Lunar and Planetary Science Conference, The Woodlands, TX, USA, 21-25 March 2016.

7. Fitzsimmons, A.; Hainaut, O.; Meech, K.J.; Jehin, E.; Moulane, Y.; Opitom, C.; Yang, B.; Keane, J.V.; Kleyna, J.T.; Micheli, M.; et al. Detection of CN gas in Interstellar Object 2I/Borisov. Astrophys. J. Lett. 2019, 885, L9. [CrossRef]

8. Jewitt, D.; Luu, J. Initial Characterization of Interstellar Comet 2I/2019 Q4 (Borisov). arXiv 2019, arXiv:1910.02547.

9. Bolin, B.T.; Lisse, C.M.; Kasliwal, M.M.; Quimby, R.; Tan, H.; Ye, Q.; Copperwheat, C.; Fernandez, Y.R.; Lin, Z.Y.; Morbidelli, A.; et al. haracterization of the Nucleus, Morphology and Activity of Interstellar comet 2I/Borisov by Optical and Near-Infrared GROWTH, Apache Point, IRTF, ZTF, Keck and HST Observations. arXiv 2019, arXiv:1910.14004.

10. De León, J.; Licandro, J.; Serra-Ricart, M.; Cabrera-Lavers, A.; Font Serra, J.; Scarpa, R.; de la Fuente Marcos, C.; de la Fuente Marcos, R. Interstellar Visitors: A Physical Characterization of Comet C/2019 Q4 (Borisov) with OSIRIS at the $10.4 \mathrm{~m}$ GTC. Res. Notes Am. Astrono. Soc. 2019, 3, 131. [CrossRef]

11. Jewitt, D.; Kim, Y.; Luu, J.; Rajagopal, J.; Kotulla, R.; Ridgway, S.; Liu, W. Episodically Active Asteroid 6478 Gault. Astrophys. J. Lett. 2019, 876, L19. [CrossRef]

12. Drilling, J.S.; Landolt, A.U. Allen's Astrophysical Quantities; Springer: Berlin, Germany, 2000; Volume 381.

13. Farnham, T.L.; Schleicher, D.G.; A'Hearn, M.F. The HB narrowband comet filters: Standard stars and calibrations. Icarus 2000, 147, 180. [CrossRef]

14. Kareta, T.; Andrews, J.; Noonan, J.W.; Harris, W.M.; Smith, N.; O’Brien, P.; Sharkey, B.N.; Reddy, V.; Springmann, A.; Lejoly, C.; et al. Carbon Chain Depletion of 2I/Borisov. arXiv 2019, arXiv:1910.03222.

15. Opitom, C.; Fitzsimmons, A.; Jehin, E.; Moulane, Y.; Hainaut, O.; Meech, K.J.; Yang, B.; Snodgrass, C.; Micheli, M.; Keane, J.V.; et al. 2I/Borisov: A C2-depleted interstellar comet. Astron. Astrophys. 2019, 631, L8. [CrossRef]

16. Tsiganis, K.; Gomes, R.; Morbidelli, A.; Levison, H.F. Origin of the orbital architecture of the giant planets of the Solar System. Nature 2005, 435, 459. [CrossRef] [PubMed]

17. Morbidelli, A.; Levison, H.F.; Tsiganis, K.; Gomes, R. Chaotic capture of Jupiter's Trojan asteroids in the early Solar System. Nature 2005, 435, 462. [CrossRef] [PubMed]

18. Gomes, R.; Levison, H.F.; Tsiganis, K.; Morbidelli, A. Origin of the cataclysmic Late Heavy Bombardment period of the terrestrial planets. Nature 2005, 435, 466. [CrossRef] [PubMed]

(C) 2019 by the author. Licensee MDPI, Basel, Switzerland. This article is an open access article distributed under the terms and conditions of the Creative Commons Attribution (CC BY) license (http:/ / creativecommons.org/licenses/by/4.0/). 